\title{
BMJ Open Occupational therapy interventions for adults with severe mental illness: a scoping review
}

\author{
María Rocamora-Montenegro, ${ }^{1}$ Laura-María Compañ-Gabucio (D) , , \\ Manuela Garcia de la Hera (id) ${ }^{1,2,3}$
}

To cite: RocamoraMontenegro M, CompañGabucio L-M, Garcia de la Hera M. Occupational therapy interventions for adults with severe mental illness: a scoping review. BMJ Open 2021;11:e047467. doi:10.1136/ bmjopen-2020-047467

- Prepublication history and additional supplemental material for this paper are available online. To view these files, please visit the journal online (http://dx.doi.org/10.1136/ bmjopen-2020-047467).

Received 02 December 2020 Accepted 15 October 2021

\section{Check for updates}

(c) Author(s) (or their employer(s)) 2021. Re-use permitted under CC BY-NC. No commercial re-use. See rights and permissions. Published by BMJ.

${ }^{1}$ Department of Public Health History of Science and Gynaecology, Universidad Miguel Hernandez de Elche, Sant Joan d'Alacant, Alicante, Spain ${ }^{2}$ ISABIAL, Instituto de Investigación Sanitaria y Biomédica de Alicante, Alicante, Comunidad Valenciana, Spain

${ }^{3}$ Consortium for Biomedical Research in Epidemiology and Public Health (CIBERESP), Madrid, Spain

Correspondence to Laura-María Compañ-Gabucio; Icompan@umh.es

\section{ABSTRACT}

Objective To identify the occupational therapy (OT) interventions in adults with severe mental illness (SMI) most investigated in intervention studies and to describe their characteristics.

Design Scoping review.

Data sources On 17 January 2020, we searched the following electronic databases: MEDLINE, Scopus, Web of Science and EMBASE. We also performed a manual search of TESEO doctoral thesis database and of the journals indexed in the first quartile of OT according to the SCImago Journal Rank. We updated our search on 10 March 2021, performing a complementary search on ProQuest database and repeating the search in all sources. The terms included in the search strategy were: schizophrenia, schizotypal personality, delusional, schizoaffective, psychotic, bipolar, major depression, obsessive-compulsive, severe mental, OT and intervention.

Study selection The study screening was peer-reviewed. Inclusion criteria were: (1) OT intervention studies in SMI: experimental, randomised, non-randomised and pilot/exploratory studies; (2) adult population with SMI: schizophrenia, schizotypal personality disorder, delusional disorder, obsessive-compulsive disorder, schizoaffective disorder, psychotic disorder, bipolar disorder, major depressive disorder; (3) OT identified as a discipline involved in the intervention; (4) English or Spanish language and (5) studies with full text available. Results Thirty-five studies met the inclusion criteria. OT interventions were classified in psychosocial, psychoeducational, cognitive and exercise interventions. The most used OT intervention was psychosocial intervention.

Conclusion Psychosocial intervention was the most investigated OT intervention in SMI, followed by psychoeducational, cognitive and exercise interventions. These interventions are usually group interventions in patients with schizophrenia, performed by a multidisciplinary team (in which an occupational therapist collaborates), with 2-3 weekly 60 min sessions and a duration of 3-6 months.

\section{INTRODUCTION}

Mental disorders represent a major issue, constituting the most frequent cause of disease burden in Europe. ${ }^{1}$ In Spain, it is estimated that at least $9 \%$ of the population is

\section{Strengths and limitations of this study}

There is little evidence regarding occupational therapy intervention in severe mental illness.

- We gave a detailed description of four types of occupational therapy intervention in severe mental illness.

- We conducted a peer-reviewed database search to ensure comprehensiveness.

- We did not assess the quality of the studies included.

- We did not include studies on addiction, anxiety or eating disorders.

affected by a mental disorder, apart from those caused by substance abuse; and slightly more than $15 \%$ will suffer from one throughout their lives. ${ }^{2}$ Severe mental illnesses (SMIs) are the most limiting mental disorders, and those with these conditions, according to the National Institute of Mental Health of the USA, are defined as 'a group of heterogeneous people, who suffer from serious psychiatric disorders that present with long-lasting mental disorders, which carry a variable degree of disability and social dysfunction, and which must be cared for through various social and health resources of the psychiatric and social care network'. ${ }^{3}$

The disorders that are included in SMI are schizophrenia, schizotypal personality disorder, delusional disorder, schizoaffective disorder, psychotic disorder, bipolar disorder, major depressive disorder and obsessivecompulsive disorder. ${ }^{4}$ Among the most frequent limitations that people with SMI experience is a lower participation in healthy activity patterns, including active and significant participation in the community, unemployment, self-care and sleep disturbances. ${ }^{56}$

Treatment for people with SMI requires, therefore, the integration of different levels of care and different interventions that include, in addition to pharmacological treatment, rehabilitation and social support 
programmes that allow them to participate in the community in a more independent and integrated way. ${ }^{7}$ One of these non-pharmacological interventions is occupational therapy $(\mathrm{OT})$, which can support recovery as a significant treatment component of these patients through meaningful activities, influencing aspects such as autonomy in activities of daily living (ADL), quality of life and personal well-being. ${ }^{8-10}$ In fact, a recent scoping review showed that different factors such as employment, may influence the recovery process of people with SMI. ${ }^{11}$ OT through vocational rehabilitation such as supported employment intervention could improve SMI patients' social functioning and hospitalisation, although not all SMI patients are motivated to work. ${ }^{12}$

Although scientific evidence regarding the OT interventions in patients with SMI is scarce, some studies suggest that these interventions have a beneficial effect. Arbesman and Logsdon ${ }^{13}$ carried out a systematic review in which they described a greater involvement in education and employment of people with SMI who were intervened with OT focused on social participation. Similarly, Conn $e t \mathrm{al}^{14}$ showed OT to be a key intervention for weight loss in people with SMI, improving their motivation and helping them to acquire healthy lifestyles.

Currently, SMI constitute a significant health problem that imposes daily limitations on those who suffer from them. In the field of OT, although there are various interventions to increase the autonomy of people with SMI and decrease their everyday restrictions, these interventions are very diverse and supported by little scientific evidence. In this sense, this scoping review is necessary to provide a detailed summary of the different OT interventions in SMI to facilitate the elaboration of evidencebased intervention programmes. Thus, we seek to answer the following research question: Which OT interventions in adults with SMI have been most investigated in intervention studies and how they are? The objective of this review was to identify the OT interventions in adults with SMI most investigated in intervention studies and to describe their characteristics.

\section{METHODOLOGY}

We performed a peer scoping review whose content was reported using the Preferred Reporting Items for Systematic Reviews and Meta-Analyses guidelines for Scoping Reviews. ${ }^{15}$ In addition, it was conducted following the indications of the Cochrane Manual ${ }^{16}$ and previously developed guidelines. ${ }^{17} 18$ Specifically, we used the Cochrane Manual to elaborate the results section and the main tables of this scoping review. We consulted the '3.4.1 Description of studies' section of chapter 3 to know how to present the main characteristics of the included studies, and the '3.4.3 Effects of interventions' section of chapter 3 to know how to present the characteristics of the OT interventions in SMI described in the included studies, adequately. As the Cochrane Manual recommendations are specific to systematic reviews, we contrasted these recommendations with those indicated in other specific scoping reviews guidelines/frameworks. ${ }^{17}{ }^{18} \mathrm{We}$ did not prepare a draft or publish a protocol for this scoping review.

\section{Search strategy and review criteria}

On 17 January 2020, we consulted the databases MEDLINE (PubMed), Scopus, Web of Science and EMBASE. These databases are widely used in review studies and the majority of them are included in the optimal database combination search ${ }^{19}$ which guarantee an adequate and efficient coverage of the scientific literature. This was supplemented by manual searching of journals indexed in the first quartile of OT according to the SCImago Journal Rank in 2018: American Journal of Occupational Therapy, Journal of Occupational Rehabilitation and Occupational Therapy Journal of Research. We excluded the Journal of Physical and Occupational Therapy in Pediatrics (POTP) as it belongs to the paediatric community, a criterion for exclusion from this review. In addition, grey literature was hand searched in TESEO which is a Spanish doctoral thesis database. We used the same search strategy in all databases and journals consulted, using all the disorders included in SMI, 'OT', and 'intervention' as search terms, with Boolean operators 'OR' and 'AND' (table 1).

In order to update and complement our search process, we consulted the Psychology Database from ProQuest on 10 March 2021. This database provides abstracts and articles from key Psychology journals, many of which are indexed in PsycINFO. In addition, we reran our search strategy in all databases and journals to identify articles published from January 2020 to March 2021.

The inclusion criteria in this review were: (1) OT intervention studies in SMI: experimental, randomised, non-randomised and pilot/exploratory studies; (2) adult population with SMI: schizophrenia, schizotypal personality disorder, delusional disorder, obsessive-compulsive disorder, schizoaffective disorder, psychotic disorder, bipolar disorder, major depressive disorder; (3) OT identified as a discipline involved in the intervention; (4) English or Spanish language and (5) studies with full text available. Those studies that did not meet the established inclusion criteria were excluded.

\section{Study selection, data extraction and synthesis}

We downloaded all titles and abstracts retrieved from all searches using Microsoft Excel. Two reviewers screened and selected the articles independently. One of them (MR-M) identified and removed duplicate records, and then two review authors (L-MC-G and MR-M) independently examined titles and abstracts and removed any irrelevant papers. Finally, L-MC-G and MR-M examined the full texts for study compliance with review eligibility criteria. A third review author (MGdIH) resolved discrepancies between these authors regarding study inclusion. We did not critically assess the quality of the included studies because it is not required in scoping review 151718 
Table 1 Database and search strategies

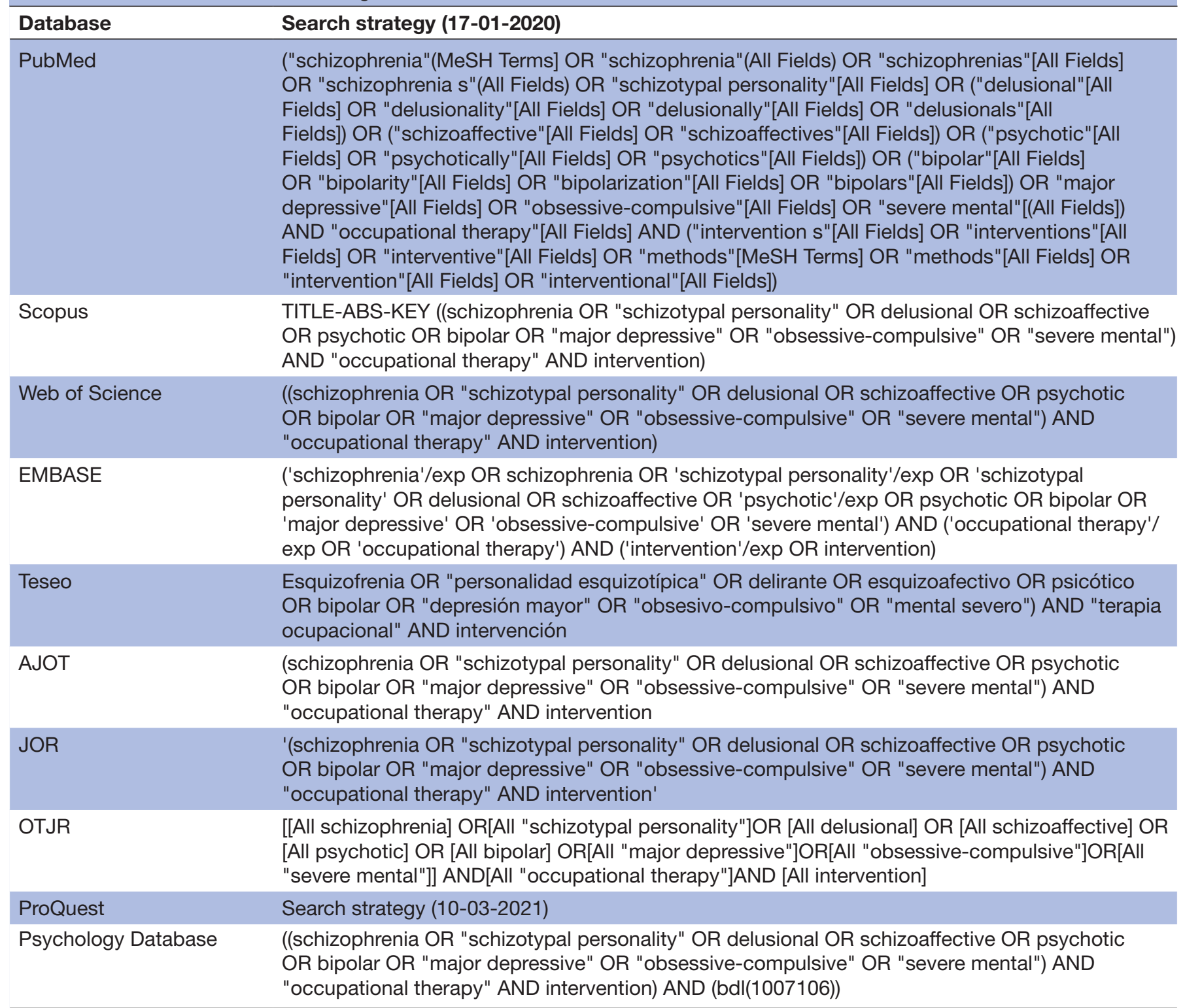

AJOT, American Journal of Occupational Therapy; JOR, Journal of Occupational Rehabilitation; OTJR, Occupational Therapy Journal of Research.

and also because our objective was not to evaluate the efficacy or effectiveness of the OT interventions in SMI. However, the main limitations found in each included study are described in online supplemental table 1 and discussed in the results section.

A data charting model and item definitions were drafted a priori by all authors. We used Microsoft Excel to create an 'Excel data form'. We conducted data extraction independently using the Excel data form and presented the characteristics of included studies following the Cochrane Manual, ${ }^{16}$ detailing author/s and year of publication, type of study, sample, OT interventions carried out, results and limitations.

We carried out a descriptive synthesis of the results. Tables and figures were used (where possible) to present the flow of study selection process and the characteristics of the included studies. In addition, as a multidisciplinary research team, we discussed the categories to classify the different types of OT interventions in SMI that are used in the included studies.

\section{Patient and public involvement}

No patients or public were involved in this review.

\section{RESULTS}

The initial search retrieved 1217 published articles on OT intervention in SMI, which resulted in 790 after removing duplicate articles. Fifty-four studies met the inclusion criteria in abstract peer review and went on to full-text review. In this initial search, we extracted data from 12 articles which fulfilled the inclusion criteria for this 


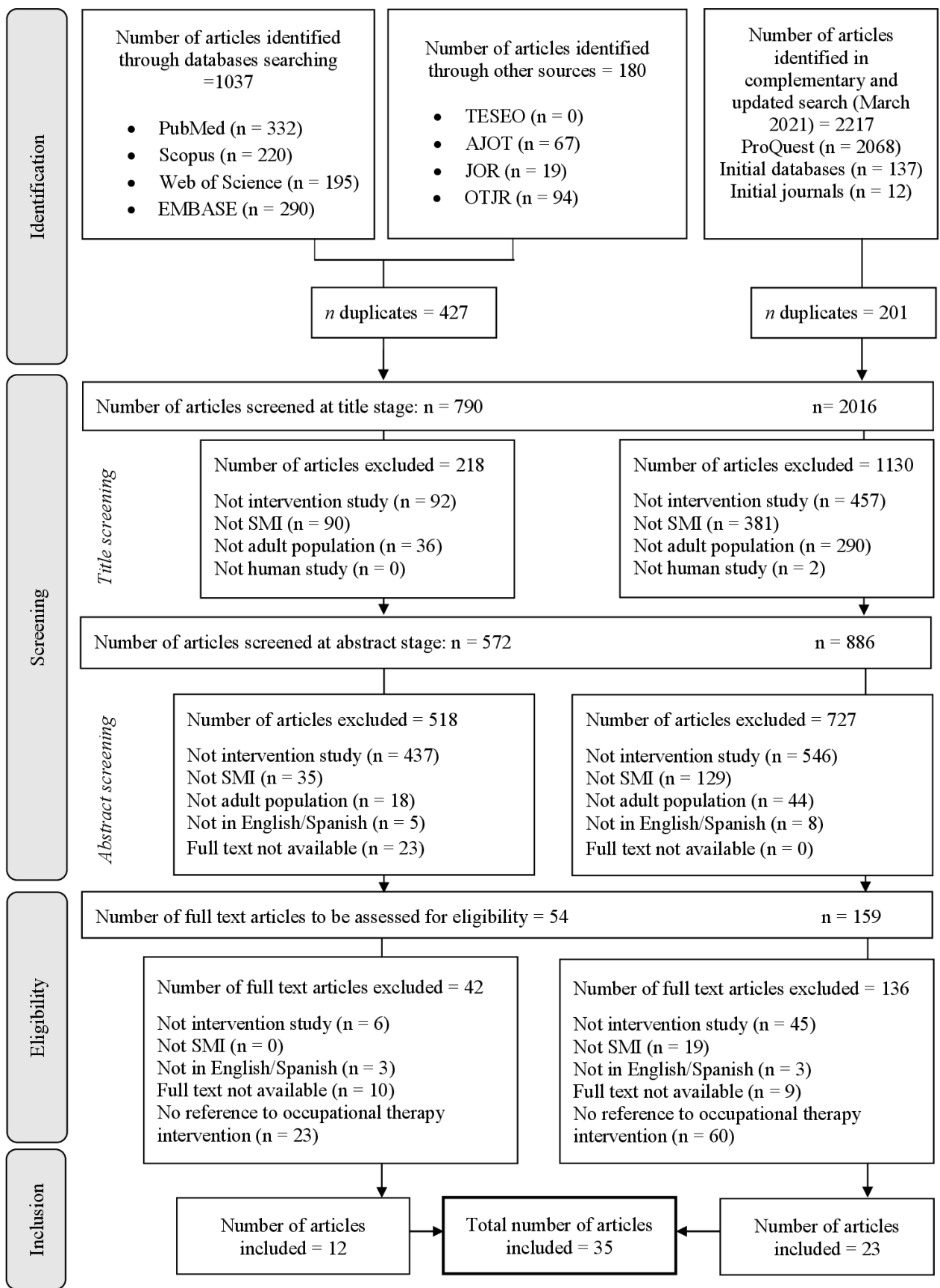

Figure 1 Flowchart of the study selection process. AJOT, American Journal of Occupational Therapy; JOR, Journal of Occupational Rehabilitation; OTJR, Occupational Therapy Journal of Research.

scoping review. The complementary search on ProQuest retrieved 2068 published articles and the updated search on initial databases and journals retrieved 149 published articles, 23 of which fulfilled the inclusion criteria. In total, we extracted data from 35 published articles on OT intervention in SMI. The study selection flowchart is shown in figure 1 .

Below we present the results regarding the characteristics of the studies included in this scoping review (online supplemental table 1) in addition to the characteristics of the OT interventions in SMI studied in the included articles (online supplemental table 2).

\section{Main characteristics of included studies}

The main characteristics of included studies are summarised in online supplemental table 1. Fifteen of the studies were carried out in Asia, 11 in Europe, 6 in North America, 1 in South America, 1 in Oceania and 1 in South Africa. Fifteen of the articles included are randomised controlled trials, ${ }^{20-34} 10$ are quasiexperimental studies, ${ }^{35-44} 5$ are a non-randomised experimental study ${ }^{45-49}$ and 5 are pilot studies. ${ }^{50-54}$

\section{OT interventions in SMI}

We present below the specific characteristics of the OT interventions in SMI to answer our research question in 
detail. The specific characteristics of included studies, such as the type of intervention, the duration of the intervention, the number of sessions performed or the measurement instruments used, are shown in online supplemental table 2.

First, we explored if the investigators used the same type of intervention in both the control and intervention groups (online supplemental table 2). In most studies $(n=18)$, standard OT intervention or pharmacological treatment was performed in both the control and intervention groups. ${ }^{20} 232426-28303233404244474850-53$ However, in the intervention group, treatment was reinforced by specific OT interventions in SMI, including: a homebased rehabilitation programme, ${ }^{20}$ a social cognition enhancement programme, ${ }^{23}$ an OT programme focused on work reintegration, ${ }^{24}$ a collaborative journal, ${ }^{26}$ a computerised cognitive programme, ${ }^{27}$ an emotion regulation skills programme, ${ }^{28}$ a group programme for balance in ADL, ${ }^{30}$ individualised $\mathrm{OT}^{32}{ }^{32}$ dance therapy, ${ }^{33}$ training in shopping skills, ${ }^{40}$ activity-based $\mathrm{OT}^{42}{ }^{4} \mathrm{OT}$ narrative medicine, ${ }^{44}$ weight loss psychiatric rehabilitation, ${ }^{47}$ metacognitive training, ${ }^{48}$ a prevocational programme ${ }^{50}$ a programme to reconnect patients with a significant activity, ${ }^{51}$ an OT programme focused on expressive activities ${ }^{52}$ and an early OT intervention. ${ }^{53}$

In the remaining studies, both the control and intervention groups participated in a different programme $(n=10),{ }^{21} 252931343638414654$ all participants received the same intervention $(n=4) 37394043$ or participants were divided in three different study groups $(n=3)$ (online supplemental table 2). ${ }^{22} 4549$ In studies with both the control and intervention groups we found interventions such as an instrumental enrichment programme versus standard OT, ${ }^{21}$ a programme focused on the management of the disease versus traditional $\mathrm{OT},{ }^{25} \mathrm{a}$ physical exercise programme versus traditional OT, ${ }^{29}$ a metacognitive programme versus traditional OT, ${ }^{31}$ a home-visit OT programme versus a management tool for daily life performance programme, ${ }^{34}$ a recovery education programme versus traditional mental health treatment, ${ }^{36}$ an aerobic dance programme versus a manual activities programme, ${ }^{38}$ a balancing everyday life programme versus traditional $\mathrm{OT}^{41}{ }^{4} \mathrm{a}$ motivational intervention versus traditional $\mathrm{OT}^{46}$ and a programme focused on executive functions versus a programme based on handmade activities. ${ }^{54}$ In the included studies with a unique study group in which all participants were treated, we found interventions such as indoor and outdoor exercise programme, ${ }^{37}$ a 'therapeutic package', ${ }^{39}$ care as usual and cognitive-behavioural therapy ${ }^{40}$ and a psychoeducation for schizophrenia programme. ${ }^{43}$ Finally, three studies included three study groups to compare two different interventions with a control group: cognitive remediation therapy versus intensive OT versus healthy patients, ${ }^{22}$ project activity group versus discussion group versus no treatment, ${ }^{45}$ and OT at the community mental health centre (CMHC) versus OT at
$\mathrm{CMHC}+$ psychosocial skill training versus outpatient follow-up. ${ }^{49}$

Second, we analysed what type of SMI was treated in each study and which was the role of the occupational therapist in the intervention team (online supplemental table 2). Schizophrenia was the most frequent object of study among the selected studies $(\mathrm{n}=25),{ }^{20-23} 252731-363840434648-54$ followed by schizoaffective disorder $(\mathrm{n}=10), 23252731323445505153$ major depression $(\mathrm{n}=6),{ }^{24} 2829343642$ a broad spectrum of disorders or non-specific SMI $(n=5)^{30} 37414447$ and bipolar disorder $(\mathrm{n}=3) .{ }^{263649} \mathrm{In}$ all the articles included, an occupational therapist formed part of the professional team, mainly as part of a multidisciplinary team composed of psychologists, nurses, dieticians, physicians, sports therapists, psychiatrics, physiotherapists, informal caregivers, pharmacists or social workers $(n=18), 20232426-33363743475054$ and secondarily alone $(\mathrm{n}=17) .2122253438-4244-46484951-53$

Third, as we have shown in online supplemental table 2 and as described below, the articles analysed were classified into four clearly differentiated interventions, except one study. ${ }^{53}$ In this study the intervention used was conventional OT in schizophrenia and schizoaffective disorder. This intervention was led exclusively by an occupational therapist, the programme lasted 12 weeks with 2-5 weekly 30 -min sessions and it included exercise, craft and daily life skills activities.

We classified the included studies in the following four interventions:

\section{Psychosocial intervention}

Psychosocial intervention was the most used OT intervention in the included studies $(\mathrm{n}=14) .202630323440-42454649505254$ In general, these interventions are performed exclusively by occupational therapists, but in five studies this intervention was performed by a multidisciplinary team made up of occupational therapists, psychologists, social workers, informal caregivers, psychiatrists or nurses. ${ }^{20} 26305054$ The main objectives of psychosocial intervention were to improve the symptoms of the disorders and occupational balance, as well as the social and work reintegration of patients with SMI. Among the different SMI treated with this intervention, psychosocial intervention was applied mainly in schizophrenia $(n=8), 2034404546495255$ schizoaffective disorder $(\mathrm{n}=4), 32344550$ bipolar disorder $(n=2),{ }^{26} 49$ in a broad spectrum of disorders $(n=2)^{3041}$ and major depressive disorder $(\mathrm{n}=2){ }^{25} 34$

The intervention programmes lasted between 3 and 9 months, and the sessions were mainly between 60 and 90 min long, although in three articles ${ }^{40} 4245$ the duration of the programme was notably shorter, lasting only 2 and 4 weeks. In turn, it should be noted that in three of the studies ${ }^{263041}$ only 1 weekly session was applied, while the rest ${ }^{32} 344042454649505254$ varied between 2 and 5 sessions per week. In one of the studies the number of session was not specify. ${ }^{20}$ This intervention was generally 
carried out in a group $(n=8),{ }^{26} 3040-42455055$ only in six studies was it carried out individually. ${ }^{203234464952}$

\section{Psychoeducational intervention}

Psychoeducational intervention was the second most used intervention in the studies included in this review $(n=9) .^{242527353639434451}$ Only in four studies, the intervention was performed exclusively by an occupational therapist. $^{25394451}$ The main objectives of this intervention were to improve disease management, to increase social abilities such as non-verbal techniques, and for the patient to acquire a significant activity, such as reading. The principal disorder treated in these interventions was schizophrenia, although in three studies were schizoaffective disorder, ${ }^{25} 51$ in one major depression ${ }^{24}$ and in one a broad spectrum of disorders. ${ }^{44}$

The intervention programmes lasted between 3 and 9 months, and the sessions were mainly between 50 and $90 \mathrm{~min}$ long, although in one article the duration of the session was $120 \mathrm{~min}^{24}$ and in other two articles the duration of the sessions was not specify. ${ }^{43} 51$ In two articles the duration of the programme was notably shorter, lasting only $2^{25}$ and 4 weeks, ${ }^{43}$ while in one article the duration of the programme was notably longer, lasting 12 months. ${ }^{36}$ In four of the studies 334344 only 1 weekly session was applied, while the rest ${ }^{2425273639}$ varied between 2 and 5 sessions per week. This intervention was generally carried out in a group $(n=7),{ }^{24} 273536394344$ only in two studies was it carried out individually. ${ }^{25} 51$

\section{Cognitive intervention}

Cognitive intervention was the third most used intervention in the studies included in this review $(n=7) .{ }^{21-2328313848}$ In four articles the intervention was carried out exclusively by an occupational therapist. ${ }^{21} 223848$ The main objective of cognitive intervention was to improve cognitive functions and processing strategies. The principal disorder treated with these interventions was schizophrenia, although in one study was it major depression. ${ }^{28}$

The duration of the intervention programmes was from 1 to 3 months, although in one of the studies the duration was 6 months. ${ }^{23}$ The sessions lasted between $45-60 \mathrm{~min}$, but in one study ${ }^{26}$ they lasted for up to 2 hours, in other they lasted ninety minutes, ${ }^{23}$ and in other the duration of the intervention programme was not specify. ${ }^{31}$ In general, in all the interventions, the sessions were carried out 2-5 times a week, except in one study ${ }^{31}$ where only 1 weekly session was applied. This intervention was generally carried out in a group $(n=5),{ }^{2328313848}$ only in two studies was it carried out individually. ${ }^{21} 22$

\section{Exercise intervention}

Less frequently, an exercise intervention was used $(n=4) .{ }^{29} 333747$ In all of these studies the intervention was carried out exclusively by a multidisciplinary team made up of occupational therapists, sport therapists, physicians, sport psychologists, psychiatrics or dieticians. The main objectives of exercise interventions were to compensate cognitive impairment common in psychiatric disabilities, to increase the knowledge and understanding of rules and to strengthen participants' ability to work as part of a team. In two studies the SMI treated was not specify, ${ }^{374}$ in one schizophrenia was treated, ${ }^{33}$ and in one major depression was treated. ${ }^{29}$

The duration of the intervention programmes was 3 months, ${ }^{3747}$ although in one of the studies the duration was 2 months, ${ }^{33}$ and in another the duration was only 1 month. ${ }^{29}$ The sessions lasted $30,{ }^{29} 40-50,{ }^{33} 60^{37}$ and 120 min. ${ }^{47}$ In general, the sessions were carried out 2-3 times a week, except in one study ${ }^{47}$ where only 1 weekly session was applied. This intervention was carried out in a group in all four studies. ${ }^{29} 333747$

Finally, we explored the measurement instruments used to assess the effect of the interventions performed in each study to facilitate the elaboration of evidence-based intervention programmes. As we have shown in online supplemental table 2, different questionnaires and scales were used. Among the included studies, the use of measuring instruments on the symptoms of the disease, mood and executive functions stands out.

\section{Symptoms of the disease}

Ten studies used Positive and Negative Symptoms Scale (PANSS) to assess the symptoms of the disease, ${ }^{27} 28$ 31-33 43 48-50 54 one used Andreasen's scale for assessment of negative symptoms and Andreasen's scale for assessment of positive symptoms,${ }^{52}$ and one used The Young Mania Rating Scale to asses maniac symptoms. ${ }^{26}$

\section{Mood}

To assess mood, that is, depression, authors used several measurement instruments and scales, such as the Montgomery Asberg Depression rating scale, the Calgary Depression Scale for Schizophrenia, the Brief Psychiatric Rating Scale, the Beck Depression Inventory or the Hamilton Depression Rating Scale. ${ }^{24}$ 26-29 313653

\section{Executive function}

In addition, investigators used a variety of measurement instruments to assess executive functions, including the Trail Making Test Parts A and B, the Brief Assessment of Cognition in Schizophrenia, the Behavioural Assessment of the Dysexecutive Syndrome, the N-Back Task and the Executive Function Performance Test. ${ }^{2022} 3238405054$

\section{Other outcomes}

To a lesser extent, other questionnaires were used to evaluate memory ${ }^{20-22} 2729384550$ such as Wechsler Adult Intelligence Scale, the General Aptitude Test Battery, Rey Auditory Verbal Learning Test, the Rey's Complex Figure or Mini-mental state examination; psychosocial functioning 213032343949 such as the Global Assessment of Functioning, the Personal and Social Performance or the Social Functioning Scale and quality of life 25273034394142 such as the 36-Item Short-Form Health Survey (SF-36) questionnaire, the General Health Questionnaire and the Manchester Short Assessment of Quality of Life. 


\section{Main results of included studies}

We summarised the main results of OT interventions in SMI in online supplemental table 1. In general, intervention groups obtained better results than control groups in all the studies, although in five of the studies included both intervention and control groups presented better results after intervention. ${ }^{24273435374043}$ Authors showed that the interventions carried out in their studies resulted in significant improvements in aspects such as participation and social functioning ( $\mathrm{n}=19),{ }^{20} 21$ 23-25 30 32-34 39 $404244-4648495154$ cognitive functioning $(n=11),{ }^{21} 22293132353640475053$ that is, executive function and memory; general symptoms $(\mathrm{n}=8))^{27-3033495052}$ and well-being $(\mathrm{n}=5),{ }^{2836374347}$ although, in three studies, these improvements were no longer presented during follow-up. ${ }^{24} 30$ In fact, it should be noted that in only three of the included studies, ${ }^{26} 3841$ the improvements found were not statistically significant.

\section{Main limitations reported in included studies}

All the studies reported limitations (online supplemental table 1). Most of the studies included in this review have a small sample size $(n=22),{ }^{20} 22-2529313337-4044454749-54$ have not evaluated the long-term effects of the intervention $(\mathrm{n}=11),{ }^{20} 24323335394245495153$ are non-blinding studies $(\mathrm{n}=10), 26273032343840424953$ have results which are not generalisable $(\mathrm{n}=7),{ }^{22333740424753}$ have a lack of randomisation $(n=5)^{3638474850}$ or they do not have a comparison group (n=6). ${ }^{33} 3537394350$

\section{DISCUSSION}

The present scoping review aimed to identify the most investigated OT interventions in adults with SMI in intervention studies and to describe their characteristics. We explored the scientific evidence available in this regard in several databases and journals, in which we found 35 articles with different types of interventions in which occupational therapists collaborated. We found four clear types of OT intervention in SMI: psychosocial, psychoeducational, cognitive and exercise interventions. The articles included in this review provide insight into the current characteristics of OT interventions in people with SMI and could provide occupational therapists with new ideas and perspectives for the implementation, development and evaluation of their interventions.

In this review, more than half $(60 \%)$ of the selected articles were published in the last decade. These results may show that although recent evidence regarding OT interventions in a mental health setting is limited, there has been an increasing number of publications related to SMI over recent years. Moreover, the oldest articles included in this review are from the year $1999^{20}{ }^{45}$ which appears to show that OT is not a relatively new healthcare discipline, but that scientific research in the field of OT has been carried out for several years. This research started very early, in fact The World Federation of Occupational Therapists meetings began in $1951,{ }^{55}$ and in some countries, like Spain, the first health department including an OT service was set up in $1969 .{ }^{56}$

In general, the included articles showed that OT intervention had beneficial results in several SMI patients' health outcomes such as cognition, social skills or mood. These positive results could be the consequence of publication bias or the consequence of the study limitations such as small sample size, lack of randomisation or non-blinded researchers, which could compromise their validity. However, the significance of the associations found in the included articles should not be influenced by these limitations. In fact, some reviews have pointed out the effectiveness of OT interventions in SMI aimed at facilitating work, ${ }^{13}$ community integration ${ }^{57}$ or weight loss. ${ }^{58}$ Moreover, OT has been identified as a non-pharmacological approach that can be an important adjunct to other psychiatric treatments. ${ }^{8}$

In this review, the most widely described OT intervention in SMI among the included studies was the psychosocial intervention followed by psychoeducational, cognitive and exercise intervention. One reason could be that psychosocial impairments should rather be seen as a consequence of chronic mental illness. ${ }^{59}$ Their improvement and a patient's greater ability to participate socially are the central treatment goals. How well this can be achieved and through which intervention must be investigated in scientific studies. Another reason could be the fact that we only included those articles where occupational therapists were one of the professionals who performed the interventions in SMI. In this sense, OT is a discipline that rehabilitates the patient through the use of occupation and meaningful activities so that they can acquire the greatest level of autonomy and daily life functioning. ${ }^{60}$ Thus, it is possible that occupational therapists use psychosocial and psychoeducational interventions more frequently than other professionals, since social limitations are not only one of the most relevant symptoms of SMI but are also closely related to an impairment in daily life functioning. ${ }^{61}$ Cognitive or exercise interventions, on the other hand, are probably performed more frequently by other professionals such as psychologist or physicians. In fact, in this review, the intervention was led exclusively by an occupational therapist in seventeen articles, nine of which were psychosocial interventions, ${ }^{32} 3440-4245464952$ and four psychoeducational interventions. ${ }^{25} 394451$

Psychosocial, psychoeducational, cognitive and exercise interventions were the main interventions that we found based on our search strategy and inclusion criteria. However, there are other interventions that can be used in SMI from OT such as vocational, individual placement and support (IPS) and place first then train interventions. ${ }^{6263}$ These interventions are usually aimed at helping people with SMI to find and maintain competitive employment as well as promote recovery and overcome barriers to participation in their jobs.$^{6264}$ An explanation for the non-inclusion of these types of interventions may be the fact that we only included those articles in which the occupational therapist was involved in the intervention 
and this was clearly specified. It would be interesting to conduct more review studies that specifically address this type of interventions.

Based on the synthesis of information on the characteristics of the interventions carried out in the articles included, we could say that a 'typical' OT programme intervention in SMI can include the following characteristics: group intervention in patients with schizophrenia, performed by a multidisciplinary team (in which an occupational therapist collaborates), with $2-3$ weekly $60 \mathrm{~min}$ sessions, and a duration of between 3 and 6 months. None of the articles contained an explanation as to why they chose these characteristics for their intervention programmes, but most of the articles mentioned that the interventions were carried out in private mental health centres, so these characteristics may be influenced by the regulations/policy of each centre. SMI symptomatology, that is, social difficulties, represents another possible factor that may influence the characteristics of the interventions; carrying out a group intervention could favour the patient's opportunities for peer contact and emotional, practical and peer support, within a safe environment for them. ${ }^{65}$

In general, regardless of the type of intervention performed in each study, the results of the articles included in this review showed positive effects of OT interventions. Psychosocial interventions resulted in improvements in the symptoms, occupational balance and sociooccupational reintegration of the patients. Other studies supported these improvements, especially of psychosocial interventions based on activity and lifestyle, and those focused on vocational and occupational rehabilitation. ${ }^{66-68}$ Psychoeducational intervention showed favourable results in these people's self-perceived health and social participation. Similarly showed Doroud et $a l^{69}$ and Petersen $e t a l,{ }^{70}$ who pointed out that participating in meaningful activities is experienced as a break from the discomfort caused by symptoms and as a means to rediscover forgotten resources and reconnect with daily life. Cognitive interventions led to improvements in memory and executive functions and consequently in SMI patients' functionality and participation. These results are in line with those found by Wykes $e t a l^{71}$ which showed that an intervention based on cognitive remediation could reduce cognitive deficits, achieving a shortterm impact on social functioning. Exercise interventions improved well-being, alertness and depression symptoms. Similar results were found in recent published studies ${ }^{72-74}$ and additionally, was found a relationship between exercise interventions and healthy lifestyles acquisition ${ }^{74}$

The measurement instruments used in the included articles to assess these outcomes varied widely between studies. Therefore, providing a synthesis of the information regarding this characteristic of the OT intervention in SMI was practically impossible for us. In general terms, PANSS was the most widely used scale among the included studies. This is consistent with the rest of the results of this scoping review if we consider that it is a specific instrument widely used to assess the presence of symptoms in schizophrenia, ${ }^{75}$ which is this the most studied type of SMI in this scoping review. Moreover, this was not the only test used to assess the illness symptoms, which were the main health outcome assessed among the included studies. Considering that psychosocial intervention was the most used intervention, we expected to find social skills as the second main health result assessed in the included studies but, instead, it was mood, that is to say, depression, followed by executive. Interestingly, mood assessment was generally performed on articles retrieved from the ProQuest psychology database (information not shown). We found that mood was one of the most studied outcomes in the included studies, and it may be partly explained by the fact that people with SMI often experience stigma which can produce consequences that can be related to low mood, such as burden, feelings of embarrassment or shame and poor quality of life.$^{76}$ In addition, people with SMI often present other chronic conditions that coexist with the SMI, ${ }^{77}$ which can also be related to mood impairment.

We highlight the implications of this review for the practice of OT and similar professionals. This scoping review provides occupational therapists with tools that facilitate the development of OT intervention sessions in SMI by knowing in advance some characteristics of these four types of intervention: psychosocial, psychoeducational, cognitive and exercise. Somehow, this updated summary of the scientific evidence that exists on SMI interventions could be useful for occupational therapists to perform evidence-based OT, although the information presented in this review should be interpreted with caution because we did not assess the quality of the included studies.

\section{Strengths and limitations}

This scoping review presents some limitations that may influence the results obtained. Although a systematic peer review was used to ensure scientific rigour, the lack of completeness of the information reported, the publication bias limiting null results intervention and selection bias are limitations for the majority of reviews. Regarding the inclusion criteria, we only included those studies published in Spanish or English and with full text available, we may, therefore, not have included significant articles because they were published in another language, this may be a potential source of bias. In addition, it was difficult to establish the search strategy because the disorders included in SMI spectrum were not clearly defined in published articles. Thus, we decided to use the WHO definition of SMI, which includes schizophrenia and related conditions, bipolar disorder and moderate and severe depression. ${ }^{78}$ This could lead to the non-inclusion of other relevant articles whose study population was other mental illnesses that could also be serious such as anxiety, addiction, personality disorders or eating disorders. Moreover, we only included in this review those articles where occupational therapists were one of the professionals who performed the interventions in SMI. Thus, 
we may not have included some articles in which occupational therapist was involved in the intervention but this was not clearly specified in the study, which favoured the selection bias. In this sense, we have not included studies in which IPS, vocational or first place then train interventions were used, which may lead to an incomplete overview of current OT interventions in SMI. Regarding the studies included in this review, it is possible that they contained biases associated with the experimental study design, which was the only type of study included in this review. In addition, we did not assess the quality of the final selected articles, so we could have included some articles with low methodological quality. However, we collected and presented the main limitations reported in included studies in an attempt to provide readers with information closely related the quality of the studies. Furthermore, not all the articles included measure the same variables or use the same measurement instruments. Although our objective was not to statistically analyse the numerical results, the great variety of measurement instruments used made difficult to compare the results between studies and to draw conclusions. Thus, the results of this scoping review must be interpreted with caution.

However, this review also has several strengths. This is a necessary and original review, because to our knowledge, there is no other review whose aim was to describe the OT interventions which are most often used in intervention studies. In addition, scoping reviews stands out for their ability to identify knowledge gaps on the subject of study, which provides opportunity for future research. This review highlights the following knowledge gaps: (1) to our knowledge, there are no OT intervention studies in SMI in Spain; (2) most of the studies had limitations that could compromises the validity of their results, such as: small sample size and lack of randomisation, (3) most of the included studies are supported by little evidence of the effects of long-term interventions; (4) there is a wide variety of measurement instruments that differ between studies and (5) there is a low representation of IPS, vocational and place first then train interventions studies in which the role of the occupational therapist was clearly specified. The results of this scoping review may provide a useful theoretical basis on which to develop new OT interventions in SMI. Especially for researchers developing interventions based on The Medical Research Council (MRC) Framework,$^{79}$ who can use the results presented in this review to complete the first stage of this framework: 'Developing complex intervention', specifically the stage 1.1 'Identifying evidence base by reviewing published literature and existing systematic reviews'. However, it would be necessary to supplement this information with the results of some systematic reviews, as indicated by the recommendations of the MRC framework.

In conclusion, the most investigated OT interventions in SMI were psychosocial, psychoeducational, cognitive and exercise interventions. These interventions are usually group interventions in patients with schizophrenia, performed by a multidisciplinary team (in which an occupational therapist collaborates), with 2-3 weekly 60 min sessions, and a duration of between 3 and 6 months. Moreover, although there are different interventions and each one covers different aspects, they all have a common objective: to reduce, through occupation, the limitations that SMI cause in patients, thus improving their quality of life. Although previous studies have shown beneficial effects of the interventions described in this review, further research is required to clearly define parameters such as optimal dose and frequency of sessions, and to understand the long-term effects of the interventions. In the case of the MRC framework, further studies are needed to continue with the stage 2 'Assessing feasibility and piloting methods'.

Acknowledgements We would like to acknowledge the English revision made by Jessica Gorlin.

Contributors All authors contributed to the conception or design of the review and to the data analysis and interpretation. MGdIH coordinated the scoping review. L-MC-G and MR-M conducted a peer-reviewed search and screening study. MR-M wrote the first draft of the paper and MGdIH and L-MC-G provided critical revision of the article. MR-M is the guarantor of this work. All authors gave final approval of the manuscript.

Funding The authors have not declared a specific grant for this research from any funding agency in the public, commercial or not-for-profit sectors.

Competing interests None declared.

Patient consent for publication Not applicable.

Provenance and peer review Not commissioned; externally peer reviewed.

Data availability statement All data relevant to the study are included in the article or uploaded as online supplemental information. No additional data available, all data relevant to the study are included in the article.

Supplemental material This content has been supplied by the author(s). It has not been vetted by BMJ Publishing Group Limited (BMJ) and may not have been peer-reviewed. Any opinions or recommendations discussed are solely those of the author(s) and are not endorsed by BMJ. BMJ disclaims all liability and responsibility arising from any reliance placed on the content. Where the content includes any translated material, BMJ does not warrant the accuracy and reliability of the translations (including but not limited to local regulations, clinical guidelines, terminology, drug names and drug dosages), and is not responsible for any error and/or omissions arising from translation and adaptation or otherwise.

Open access This is an open access article distributed in accordance with the Creative Commons Attribution Non Commercial (CC BY-NC 4.0) license, which permits others to distribute, remix, adapt, build upon this work non-commercially, and license their derivative works on different terms, provided the original work is properly cited, appropriate credit is given, any changes made indicated, and the use is non-commercial. See: http://creativecommons.org/licenses/by-nc/4.0/.

\section{ORCID iDs}

Laura-María Compañ-Gabucio http://orcid.org/0000-0001-5324-1535

Manuela Garcia de la Hera http://orcid.org/0000-0001-5742-2704

\section{REFERENCES}

1 Instituto Nacional de Epidemiología de Salud Carlos III. Salud mental Y Salud Pública en España: Vigilancia epidemiológica, 2017. Available: http://publicaciones.isciii.es [Accessed 20 Nov 2019].

2 World Health Organization. International classification of impairments, disabilities and handicaps. Geneva: WHO, 1980.

3 National Institute of Mental Health (NIMH). Towards a model for a comprehensive community based mental health system, 1987. Available: https://www.nih.gov/about-nih/what-we-do/nih-almanac/ national-institute-mental-health-nimh [Accessed 20 Nov 2019].

4 World Health Organization. The ICD-10 classification of mental and behavioral disorders. Clinical descriptions and diagnostic guidelines. WHO, 1992. 
5 Lipskaya-Velikovsky L, Elgerisi D, Easterbrook A, et al. Motor skills, cognition, and work performance of people with severe mental illness. Disabil Rehabil 2019;41:1396-402.

6 Swarbrick M, Noyes S. Effectiveness of occupational therapy services in mental health practice. Am J Occup Ther 2018;72:7205170010p1-7205170010.

7 Diez-Carral B, Montoya-del-Corte P, Solana-Álvarez A. La atención sociosanitaria a personas Con trastorno mental Grave: Una propuesta de red de dispositivos para Cantabria, 2015. Available: https://www.ascasam.org/images/interes/TesinaMAGDS.pdf [Accessed 28 November 2019].

$8 \mathrm{Höhl} \mathrm{W,} \mathrm{Moll} \mathrm{S,} \mathrm{Pfeiffer} \mathrm{A.} \mathrm{Occupational} \mathrm{therapy} \mathrm{interventions} \mathrm{in} \mathrm{the}$ treatment of people with severe mental illness. Curr Opin Psychiatry 2017;30:300-5

9 Lindström M, Hariz G-M, Bernspång B. Dealing with real-life challenges: outcome of a home-based occupational therapy intervention for people with severe psychiatric disability. OTJR 20122012;32:5-14

10 Brown C, Stoffel V. Occupational therapy in mental health: a vision for participation. 2nd ed. Philadelphia: FA Davis, 2019.

11 Bitter N, Roeg D, van Nieuwenhuizen C, et al. Recovery in supported Accommodations: a scoping review and synthesis of interventions for people with severe mental illness. Community Ment Health J 2020;56:1053-76.

12 Oka M, Otsuka K, Yokoyama N, et al. An evaluation of a hybrid occupational therapy and supported employment program in Japan for persons with schizophrenia. Am J Occup Ther 2004:58:466-75

13 Arbesman M, Logsdon DW. Occupational therapy interventions for employment and education for adults with serious mental illness: a systematic review. Am J Occup Ther 2011;65:238-46.

14 Conn A, Bourke N, James C, et al. Occupational therapy intervention addressing weight gain and obesity in people with severe mental illness: a scoping review. Aust Occup Ther J 2019;66:446-57.

15 Tricco AC, Lillie E, Zarin W, et al. PRISMA extension for scoping reviews (PRISMA-ScR): checklist and explanation. Ann Intern Med 2018;169:467-73.

16 Higgins J, Green S. Cochrane Handbook for systematic reviews of interventions. The Cochrane collaboration, 2011. Available: http:// handbook.cochrane.org/ [Accessed 16 February 2020].

17 Arksey H, O'Malley L. Scoping studies: towards a methodological framework. Int J Soc Res Methodol 2005;8:19-32.

18 Levac D, Colquhoun H, O'Brien KK. Scoping studies: advancing the methodology. Implement Sci 2010;5:69.

19 Bramer WM, Rethlefsen ML, Kleijnen J, et al. Optimal database combinations for literature searches in systematic reviews: a prospective exploratory study. Syst Rev 2017;6:245.

20 Sellwood W, Thomas CS, Tarrier N, et al. A randomised controlled trial of home-based rehabilitation versus outpatient-based rehabilitation for patients suffering from chronic schizophrenia. Soc Psychiatry Psychiatr Epidemiol 1999;34:250-3.

21 Hadas-Lidor N, Katz N, Tyano S, et al. Effectiveness of dynamic cognitive intervention in rehabilitation of clients with schizophrenia. Clin Rehabil 2001;15:349-59.

22 Wykes T, Brammer M, Mellers J, et al. Effects on the brain of a psychological treatment: cognitive remediation therapy: functional magnetic resonance imaging in schizophrenia. $\mathrm{Br} \mathrm{J}$ Psychiatry 2002;181:144-52.

23 Choi K-H, Kwon J-H. Social cognition enhancement training for schizophrenia: a preliminary randomized controlled trial. Community Ment Health J 2006;42:177-87.

24 Schene AH, Koeter MWJ, Kikkert MJ, et al. Adjuvant occupational therapy for work-related major depression works: randomized trial including economic evaluation. Psychol Med 2007;37:351-62.

25 Chan SH-W, Lee SW-K, Chan IW-M. Trip: a psycho-educational programme in Hong Kong for people with schizophrenia. Occup Ther Int 2007;14:86-98.

26 Castle D, White C, Chamberlain J, et al. Group-Based psychosocial intervention for bipolar disorder: randomised controlled trial. $\mathrm{Br} \mathrm{J}$ Psychiatry 2010;196:383-8.

27 Jahn T, Pitschel-Walz G, Gsottschneider A, et al. Neurocognitive prediction of illness knowledge after psychoeducation in schizophrenia: results from the Munich COGPIP study. Psychol Med 2011;41:533-44.

28 Berking $\mathrm{M}$, Ebert $\mathrm{D}$, Cuijpers $\mathrm{P}$, et al. Emotion regulation skills training enhances the efficacy of inpatient cognitive behavioral therapy for major depressive disorder: a randomized controlled trial. Psychother Psychosom 2013;82:234-45.

29 Buschert V, Prochazka D, Bartl H, et al. Effects of physical activity on cognitive performance: a controlled clinical study in depressive patients. Eur Arch Psychiatry Clin Neurosci 2019;269:555-63.
30 Eklund M, Tjörnstrand C, Sandlund M, et al. Effectiveness of balancing everyday life (BEL) versus standard occupational therapy for activity engagement and functioning among people with mental illness - a cluster RCT study. BMC Psychiatry 2017;17:1-12.

31 Pos K, Meijer CJ, Verkerk O, et al. Metacognitive training in patients recovering from a first psychosis: an experience sampling study testing treatment effects. Eur Arch Psychiatry Clin Neurosci 2018;268:57-64

32 Shimada T, Ohori M, Inagaki Y, et al. A multicenter, randomized controlled trial of individualized occupational therapy for patients with schizophrenia in Japan. PLoS One 2018;13:e0193869-18.

33 Gökcen A, Ekici G, Abaoğlu $\mathrm{H}$, et al. The healing effect of goal-oriented dance and movement therapy in schizophrenia: a rater-blinded randomized controlled trial. Arts Psychother 2020;71:101702.

34 Mashimo I, Yotsumoto K, Fujimoto $\mathrm{H}$, et al. Effects of Homevisit occupational therapy using a management tool for daily life performance on severe mental illness: a multicenter randomized controlled trial. Kobe J Med Sci 2020;66:E119-28.

35 Mclnnis E, Sellwood W, Jones C. A cognitive behavioural groupbased educational programme for psychotic symptoms in a low secure setting: a pilot evaluation. British Jnl Forensic Practice 2006;8:36-46.

36 Dunn EC, Sally Rogers E, Hutchinson DS, et al. Results of an innovative university-based recovery education program for adults with psychiatric disabilities. Adm Policy Ment Health 2008:35:357-69.

37 Tetlie T, Eik-Nes N, Palmstierna T, et al. The effect of exercise on psychological \& physical health outcomes: preliminary results from a Norwegian forensic hospital. J Psychosoc Nurs Ment Health Serv 2008; $46: 38-43$

38 Chen M-D, Kuo Y-H, Chang Y-C, et al. Influences of aerobic dance on cognitive performance in adults with schizophrenia. Occup Ther Int 2016;23:346-56.

39 Singh U, Singh B, et al. Assessment and management of SocioOccupational functioning of persons with chronic schizophrenia: effect of social skills training. JPR 2018;13:189-98.

$40 \mathrm{Kim}$ Y-S, Park J-H, Lee S-A. Is a program to improve groceryshopping skills clinically effective in improving executive function and instrumental activities of daily living of patients with schizophrenia? Asian J Psychiatr 2020;48:101896.

41 Argentzell E, Bäckström M, Lund K, et al. Exploring mediators of the recovery process over time among mental health service users, using a mixed model regression analysis based on cluster RCT data. BMC Psychiatry 2020;20:520

42 Ramano EM, de Beer M. The outcome of two occupational therapy group programs on the social functioning of individuals with major depressive disorder. Occupational Therapy in Mental Health 2020;36:29-54

43 Shinozaki A, Hayashi T, Okamura H. Effects of a Psychoeducation program for people with schizophrenia aimed at increasing subjective well-being and the factors influencing those effects: a preliminary study. Psychiatr Q 2020;91:45-52.

44 Wasmuth S, Wilburn VG, Hamm JA, et al. Comparing NarrativeInformed occupational therapy in adult outpatient mental health to treatment as usual: a quasi-experimental feasibility study with preliminary treatment outcomes. Occupational Therapy in Mental Health 2021;37:56-71.

45 Schindler VP. Group effectiveness in improving social interaction skills. Psychiatr Rehabil J 1999;22:349-54.

46 CY W. Facilitating intrinsic motivation in individuals with psychiatric illness: a study on the effectiveness of an occupational therapy intervention. OTJR 2001;21:142-67.

47 Brown C, Goetz J, Van Sciver A, et al. A psychiatric rehabilitation approach to weight loss. Psychiatr Rehabil J 2006;29:267-73.

48 Kaizerman-Dinerman A, Roe D, Josman N. An efficacy study of a metacognitive group intervention for people with schizophrenia. Psychiatry Res 2018;270:1150-6.

49 Karaman İmran Gökçen Yilmaz, Kasal Meltem İzcı, İngeç C, et al. Effect of adjunct psychosocial skills training on social functioning of schizophrenia patients who get occupational therapy in a community mental health center: a comparative study. Noro Psikiyatr Ars 2020;57:248-53.

50 Rouleau S, Saint-Jean M, Stip E, et al. The impact of a prevocational program on cognition, symptoms, and work reintegration in schizophrenia. Occupational Therapy in Mental Health 2009;25:26-43.

51 Edgelow M, Krupa T. Randomized controlled pilot study of an occupational time-use intervention for people with serious mental illness. Am J Occup Ther 2011;65:267-76. 
52 Foruzandeh N, Parvin N. Occupational therapy for inpatients with chronic schizophrenia: a pilot randomized controlled trial. Jpn J Nurs Sci 2013;10:136-41

53 Tanaka C, Yotsumoto K, Tatsumi E, et al. Improvement of functional independence of patients with acute schizophrenia through early occupational therapy: a pilot quasi-experimental controlled study. Clin Rehabil 2014:28:740-7.

54 Vizzotto ADB, Celestino DL, Buchain PC, et al. A pilot randomized controlled trial of the occupational goal intervention method for the improvement of executive functioning in patients with treatmentresistant schizophrenia. Psychiatry Res 2016;245:148-56.

55 World Federation of Occupational Therapists (WFOT). History. Available: https://wfot.org/about/history [Accessed 30 March 2021].

56 Triviñó Juárez JM, Romero Ayuso DM. Institucionalización de la terapia ocupacional en España. In: Romero Ayuso DM, Moruno Miralles P, eds. Terapia ocupacional: Teoría Y técnicas. Barcelona: Masson, 2003: 29-40.

57 Gibson RW, D'Amico M, Jaffe L, et al. Occupational therapy interventions for recovery in the areas of community integration and normative life roles for adults with serious mental illness: a systematic review. Am J Occup Ther 2011;65:247-56.

58 Naslund JA, Whiteman KL, McHugo GJ, et al. Lifestyle interventions for weight loss among overweight and obese adults with serious mental illness: a systematic review and meta-analysis. Gen Hosp Psychiatry 2017;47:83-102.

59 Gühne U, Weinmann S, Riedel-Heller SG, et al. Psychosocial therapies in severe mental illness: update on evidence and recommendations. Curr Opin Psychiatry 2020;33:414-21.

60 Krupa T, Fossey E, Anthony WA, et al. Doing daily life: how occupational therapy can inform psychiatric rehabilitation practice. Psychiatr Rehabil J 2009;32:155-61.

61 Viertiö S, Tuulio-Henriksson A, Perälä J, et al. Activities of daily living, social functioning and their determinants in persons with psychotic disorder. Eur Psychiatry 2012;27:409-15.

62 Roeg D, de Winter L, Bergmans C, et al. iPS in supported housing: fidelity and employment outcomes over a 4 year period. Front Psychiatry 2020;11:622061.

63 Prior S, Maciver D, Aas RW, et al. An enhanced individual placement and support (iPS) intervention based on the model of human occupation (MOHO); a prospective cohort study. BMC Psychiatry 2020;20:36

64 Akasyah W, Santosa WRB. Vocational rehabilitation based recovery of patients with mental disorders (ODGJ). Sjik 2020;9:743-50.

65 Morant N, Davidson M, Wackett J, et al. Acute day units for mental health crises: a qualitative study of service user and staff views and experiences. BMC Psychiatry 2021;21:146.
66 Hoshii J, Yotsumoto K, Tatsumi E, et al. Subject-chosen activities in occupational therapy for the improvement of psychiatric symptoms of inpatients with chronic schizophrenia: a controlled trial. Clin Rehabil 2013;27:638-45.

67 Lambert RA, Lorgelly P, Harvey I, et al. Cost-effectiveness analysis of an occupational therapy-led lifestyle approach and routine general practitioner's care for panic disorder. Soc Psychiatry Psychiatr Epidemiol 2010;45:741-50.

68 Poon MYC, Siu AMH, Ming SY. Outcome analysis of occupational therapy programme for persons with early psychosis. Work 2010;37:65-70

69 Doroud N, Fossey E, Fortune T. Recovery as an occupational journey: a scoping review exploring the links between occupationa engagement and recovery for people with enduring mental health issues. Aust Occup Ther J 2015;62:378-92.

70 Petersen KS, Bjørkedal STB, Torsting AM, et al. Occupational therapy interventions in mental health: a scoping review of recent evidence. Int J Ther Rehabil 2019;26:1-21.

71 Wykes T, Reeder C, Corner J, et al. The effects of neurocognitive remediation on executive processing in patients with schizophrenia. Schizophr Bull 1999:25:291-307.

$72 \mathrm{Ng}$ SSW, Leung TKS, Ng PPK, et al. Activity participation and perceived health status in patients with severe mental illness: a prospective study. East Asian Arch Psychiatry 2020;30:95-100.

73 Ringen PA, Falk RS, Antonsen B, et al. Using motivational techniques to reduce cardiometabolic risk factors in long term psychiatric inpatients: a naturalistic interventional study. BMC Psychiatry 2018;18:255

74 Korman N, Fox H, Skinner T, et al. Feasibility and acceptability of a student-led lifestyle (diet and exercise) intervention within a residential rehabilitation setting for people with severe mental illness, go heart (group occupation, health, exercise and rehabilitation treatment). Front Psychiatry 2020;11:319.

75 Peralta V, Cuesta MJ. Psychometric properties of the positive and negative syndrome scale (PANSS) in schizophrenia. Psychiatry Res 1994:53:31-40.

76 Javed A, Lee $\mathrm{C}$, Zakaria $\mathrm{H}$, et al. Reducing the stigma of mental health disorders with a focus on low- and middle-income countries. Asian J Psychiatr 2021;58:102601.

77 Stein DJ, Benjet C, Gureje O, et al. Integrating mental health with other non-communicable diseases. BMJ 2019;364:1295.

78 World Health Organization. Helping people with severe mental disorders live longer and healthier lives: policy brief. WHO, 2017.

79 Craig P, Dieppe P, Macintyre S, et al. Developing and evaluating complex interventions: the new medical Research Council guidance. BMJ 2008;337:a1655. 